\title{
Delayed Axillary Nerve Motor Conduction Latency and Reduced CMAP Amplitudes in the Paretic Limbs of Patients With a Cerebrovascular Episode
}

\author{
Bharati Taksande a, b, Minal Ambade ${ }^{a}$, Rajnish Joshi ${ }^{a}$
}

\begin{abstract}
Background: To see for Nerve conduction changes in the axillary nerve in the patients of stroke.

Methods: All eligible and consenting patients of stroke underwent electrophysiology studies of both axillary nerves. The parameters which are looked for the measurement of neuropathy are Motor latency, CMAP (Compound muscle action potential), SNAP (sensory nerve action potential) and the conduction velocity.
\end{abstract}

Results: CMAP amplitudes were significantly lower, and CML was significantly higher among paretic limbs as compared to nonparetic limb, ie the axillary nerve in the paretic limb gets affected in the patients of stroke.

Conclusions: The study is to prove the probability of axillary nerve injury after shoulder subluxation due to stroke and to improve preventive and rehabilitative measures as even in cases of complete recovery, the disability remains.

Keywords: Stroke; Axillary nerve; Nerve conduction studies

\section{Introduction}

Cerebrovascular episode (commonly known as stroke) is a sudden onset neurological deficit, due to compromised blood

Manuscript accepted for publication October 27, 2011

${ }^{\mathrm{a}}$ Department of Medicine, MGIMS, Sewagram, Maharashtra, India

${ }^{\mathrm{b}}$ Corresponding author: Bharati Taksande, Associate professor, JNMC, Sawangi, Wardha. Email: bharati.taksande@gmail.com

doi:10.4021/jnr72e supply to a part of cerebrum or brain-stem. It is a common cause of hospitalization, and is third most common cause of death and disabling neurological damage. Worldwide about 15 million individuals have a stroke and about a one third of them die [1]. Usual presentation of stroke is hemiparesis, and depending upon the severity of disease the muscle power in muscle groups in the paretic limb may range from grade 0 (no movement of the paretic limb) to grade 4 (restricted movement against resistance) according to medical research council (MRC) grading for motor power. According to American heart Association (AHA) stroke guidelines, limb physiotherapy should be initiated early after onset of stroke to enhance recovery in motor power [2]. In India stroke rehabilitation often gets neglected, despite the fact that it is only modality which can improve motor function in the paretic limbs.

Although cerebrovascular episode does not cause peripheral nerve injury, there are theoretical reasons to believe that axillary nerve may get injured in patients with stroke related hemiparesis. Axillary nerve (a branch of posterior cord of brachial plexus) passes below the shoulder joint, before supplying two muscles of shoulder girdle-deltoid and teres minor. It has a very small sensory supply to shoulder joint and skin over the shoulder [3]. Limb paresis due to cerebrovascular episode affects all shoulder muscles, and can lead to inferior subluxation of humeral head from glenoid cavity. Continuous traction due to subluxation may injure the axillary nerve winding around the surgical neck of humerus. Such an injury may either be a demyelination or axonopathy [4]. This secondary peripheral nerve injury can go unnoticed, as it is not possible to clinically distinguish if shoulder muscles are week due to stroke or axillary nerve injury.

Since axillary nerve is predominantly motor in its supply, motor conduction latency (MCL) and compound motor action potential (CMAP) will be a measure of electrical activity in this nerve. If axillary nerve indeed gets injured, it should cause prolongation of MCL and a reduction in CMAP amplitudes. In our extensive literature search we found only one previous study which suggested changes in electro-physiological properties in axillary nerve in a paretic limb. Since the axillary nerve injury hypothesis in patients with stroke is largely unproven, we planned a pilot study to determine 
if evidence of axillary nerve injury (in form of prolongation of MCL and a reduction in CMAP amplitudes) is seen in the paretic limb, as compared to the non-paretic limb in patients with cerebrovascular episode.

\section{Methods}

\section{Setting}

The study was conducted at Mahatma Gandhi Institute of Medical Sciences, Sevagram a rural teaching hospital in Maharashtra. Of more than 10,000 admissions to the medicine wards of the hospital, about 200 are due to stroke, and one-fourth of them would have a mortality [5]. Usually patients with a moderately disabling stroke follow up in the out-patient department of the hospital. This is so because most mild strokes would recover, and those survivors with a severe stroke experience high transportation costs for coming to the hospital. We conducted our study in the outpatient setting, where most stroke survivors present for seeking care.

\section{Patients, inclusion and exclusion criteria}

We screened all patients presenting to medicine outpatient department, to identify those with stroke survivors who had: 1) Stroke within the preceding three month period; 2) Had a flaccid paralysis of the affected limb (MRC grade less than 3 at the time of stroke. We excluded patients who had: 1) More than one stroke episodes in the past; 2) Had a past history of trauma to the affected limb or past known previous nerve injury of either limb. We approached all patients who satisfied inclusion and exclusion criteria for participation in the study and sought a written informed consent for participation. All eligible and consenting patients were included in the study.

The paretic limbs of the included patients were classified as "case" limbs, while the non-paretic limbs were classified as "controls". Thus each patient was its own control. Such a study design ensured that a number of potential confounders which could affect neuronal electrophysiology (such as smoking status, diabetes mellitus, dyslipidemia, concurrent drug intake etc.) will be equally distributed for case and control limbs, and any change in MCL or CMAP can only be attributed to limb paresis.

\section{Study procedures}

All eligible and consenting patients underwent electrophysiology studies of both axillary nerves. This electro diagnostic test was performed in the department of neurophysiology laboratory in the department of physiology, on RMS EMGII Machine. The temperature of the laboratory was maintained to 22 - 23 degree Celsius. For axillary nerve electrophysiology, recording electrode was placed on the most prominent

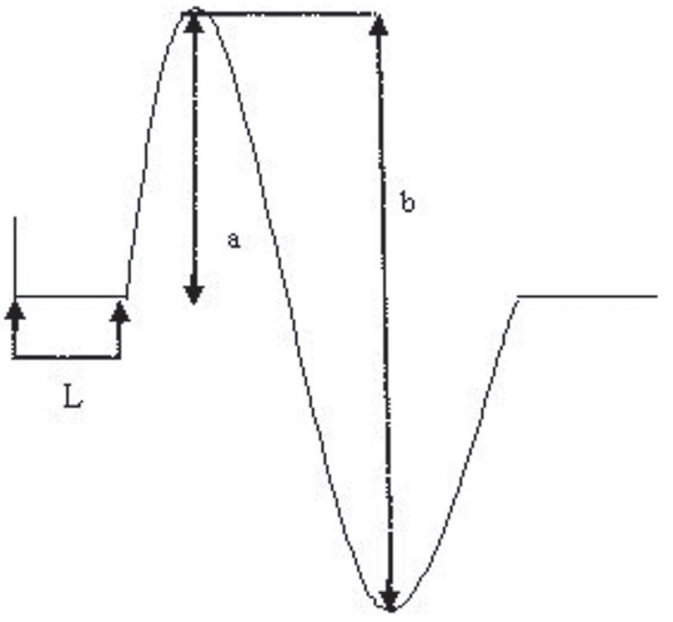

Figure 1. Measurement of CMAP latency and amplitude. $\mathrm{L}=$ latency; $c$ = base to peak amplitude; $b=$ peak to peak amplitude.

portion of middle of the deltoid with reference over its insertion. Surface stimulation was performed at the Erbs point (supraclavicular fossa). Ground electrode was placed on the acromion process. Electrical nerve stimulation of 500 Volts, well tolerated by the patients, was given at the Erb's point, slightly above the upper margin of the clavicle and lateral to the clavicular head of the sternocleidomastoid muscle. Stimulator pulse duration of the square wave was 0.1 .

The parameters which are looked for the measurement of neuropathy are Motor latency, CMAP (Compound muscle action potential), SNAP (sensory nerve action potential) and the conduction velocity. There is no conduction technique to evaluate directly sensory component of axillary nerve. Therefore, the measurement of nerve conduction in the axillary nerve included the motor latency and amplitude of CMAP.

The onset latency is the time in milliseconds from the stimulus artifact to the first negative deflection of CMAP as shown in the Figure 1 below. The onset latency is the measure of conduction in the fastest conducting motor fibers. It also includes neuromuscular transmission time and the propagation time along the muscle membrane which constitute the residual latency. The amplitude of CMAP is measured from base line to the negative peak (base to peak) or between negative and positive peaks (peak to peak). The amplitude of CMAP in our was measured from negative to positive peaks that is peak to peak. The amplitude correlates with the number of nerve fibers. The unit of measurement of CMAP is in microvolt. Motor nerve conduction velocity is calculated by measuring the distance in millimeter between two points of stimulation, which is divided by the latency difference in millisecond. However, nerve conduction velocity is not calculated in the axillary nerve as it has only one point of stimulation. Hence in this study we measured CML and CMAP in the affected as well as unaffected limbs as a continuous 
Table 1. Baseline Characteristics of Included Patients

\begin{tabular}{lcccc}
\hline & Mean & Standard deviation & Median & Inter quartile range \\
\hline Age (years) & 58 & 4.1 & 59 & $46-65$ \\
Stroke duration (days) & 57.9 & 11.39 & 56 & $44-76$ \\
$\begin{array}{l}\text { Muscle power in shoulder abductors } \\
\text { (MRC grade) }\end{array}$ & 3 & 1.37 & - & - \\
\hline
\end{tabular}

measure.

\section{Statistical analysis}

We performed a descriptive analysis of the baseline parameters for the included patients, and expressed the distribution of variables as median and range. We compared the distribution of key outcome variables (CML, and CMAP) in paretic and non-paretic limb using two-tailed student's ttest. Further we classified CML as prolonged if more than $3.5 \mathrm{msec}$, and CMAP as reduced if less than $50 \%$ than that in the opposite limb. These categorical variables were compared between paretic and non-paretic limb using chi square test of significance. We a priori considered a $\mathrm{P}$ value of less than 0.01 as significant both for student's t-test and chi-test statistics.

\section{Results}

We included a total of 21 patients (42 limbs) in the study between 1st August and 31st August 2010. All patients were right handed, 9 were men (42.86\%), and 12 women (57.14\%). Of the patients in study 13 had a thrombotic stroke, while remaining had a hemorrhagic stroke. The baseline parameters of the included patients are as shown in the Table 1 below.

CMAP amplitudes was significantly lower, and CML was significantly higher among paretic limbs as compared to non-paretic limb (Table 2)

Of 21 paretic limbs 13 (61\%) had CML duration of 3.5 msec or more. None of the axillary nerves in the non-paretic limbs had CML duration above this cut-off.

The graph (Fig. 2) shows the distribution of Motor Latency in the paretic and the non paretic limb with median of 3.54 and inter quartile range of $2.69-3.9$ in the paretic limb and median of 3.05 and inter quartile range of $2.8-3.34$ in the non paretic limb.

The graph (Fig. 3) shows the distribution of CMAP in the paretic and the non paretic limb with median of 10.51 and inter quartile range of $13-16.2$ in the paretic limb and median of 14.63 and inter quartile range of $7.9-13.1$ in the non paretic limb.

\section{Discussion}

In this study we found that CMAP amplitudes are significantly reduced and CML duration is significantly increased in axillary nerve of the paretic limbs as compared to the non-paretic limb. These findings suggest that axillary nerve electrophysiology is altered in paretic limb in patients with stroke. A likely cause of this finding is axillary nerve injury in the paretic limb due to subluxation of the glenohumeral joint.

Subluxation of the glenohumeral joint is a well-recognized complication experienced by stroke patients. The reported incidence of shoulder subluxation varies greatly, from $17 \%$ to $81 \%[6,7]$. Shoulder subluxation is a partial dislocation of the arm at the shoulder joint that occurs in many people after a stroke. In most cases the humerus (upper arm bone) drops down out of the shoulder joint. This condition is caused by either extreme muscle weakness around the shoulder, or spastic muscles. Axillary nerve injury remains the

Table 2. Axillary Nerve Electrophysiology Parameters in Paretic and Non-Paretic Limb

\begin{tabular}{ccccccc}
\hline & \multicolumn{2}{c}{ Paretic limb } & & Non-paretic limb & P value \\
\hline Variable & Mean (SD) & Median (Range) & Mean (SD) & Median (Range) & \\
CMAP & $10.51(0.90)$ & $14.8(13-16.2)$ & $14.63(1.64)$ & $10.4(7.9-13.1)$ & $<0.001$ \\
CML & $3.54(0.38)$ & $3.67(2.69-3.9)$ & $3.05(0.21)$ & $3.02(2.8-3.3)$ & $<0.001$ \\
\hline
\end{tabular}




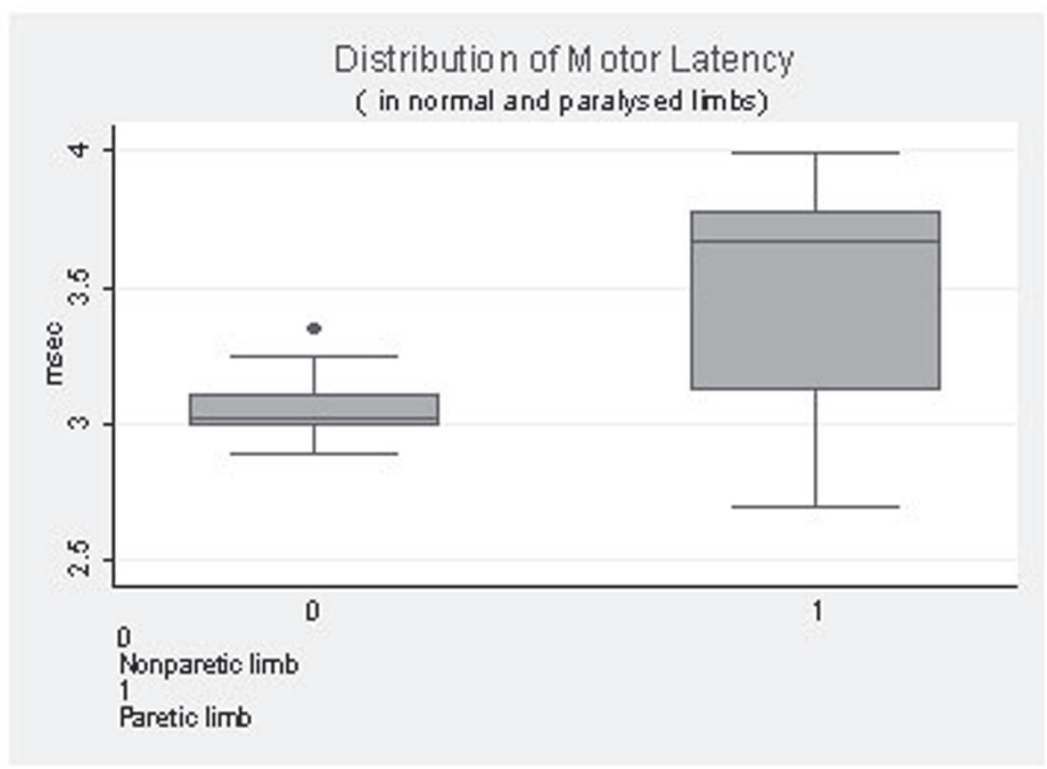

Figure 2. Distribution of motor latency in normal and paralysed limbs.

most common peripheral nerve injury to affect the shoulder. It most often is seen after glenohumeral joint dislocation, proximal humerus fracture, or a direct blow to the deltoid muscle [8]. Inferior glenohumeral joint displacement, referred to as shoulder subluxation, is one of the most common musculoskeletal problems of the upper limb in individuals after stroke. Without muscle activity, the gravitational pull on the humerus causes stretching of the capsule of the shoulder joint resulting in shoulder subluxation [9]. Teasell suggested that other factors contributing to subluxation include improper positioning, lack of support in the upright position, and pulling on the hemiplegic arm when transferring the patient [10].

The muscular tone in the paralysed upper limb of our patients remained flaccid for more than several weeks. Ring et al. [11] found that found that among 6 stroke patients that manifested certain deterioration of their gleno-humeral alignment, 5 had an electromyographic feature of axillary nerve damage. The most common zone of injury is just proximal to the quadrilateral space. Ring et al suggested that downward subluxation is able to produce traction on the axillary nerve as it winds around the surgical neck of the humeral shaft.

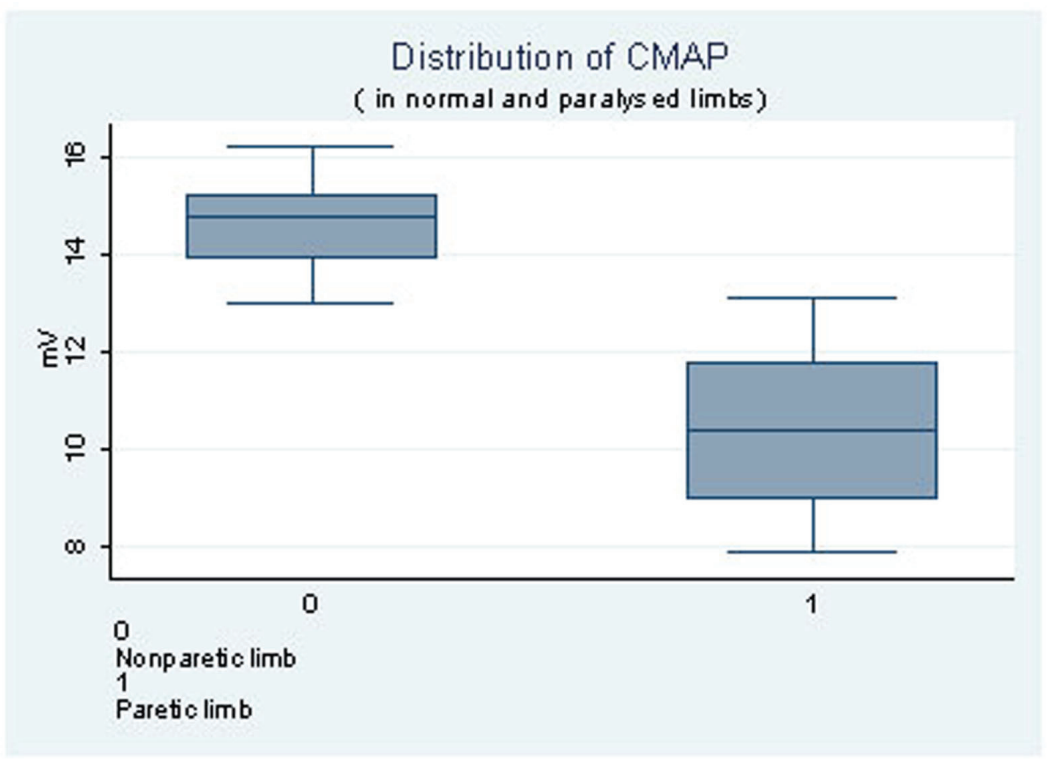

Figure 3. Distribution of CMAP in normal and paralysed limbs. 
But gentle range of motion and functional, cyclical, electrical stimulation of shoulder muscles can prevent or reduce shoulder subluxation in hemiplegics with shoulder pain. But prevention and rehabilitation is usually neglected.

Some researchers believe that in stroke, hemiparesis occurs which leads to shoulder muscle power loss. Injury to the axillary nerve may cause reduction in nerve conduction velocity. However, axillary nerve injury has not been reported. Extensive literatures seemingly reveal only one study regarding the same. So, if occurs injury to axillary nerve, then among patients with cerebrovascular accidents, is the nerve conduction velocity reduced in hemiparetic limb as compared to unaffected limb? If injury leads to reduction in nerve conduction velocity then preventive or rehabilitative measures can be taken. Since, stroke occurs suddenly, even if revascularization takes place and recovery occurs, shoulder muscles may not recover leading to subluxation and axillary nerve injury. Therefore, preventive and rehabilitative measures necessarily should be taken. The study is to prove the probability of axillary nerve injury after shoulder subluxation due to stroke and to improve preventive and rehabilitative measures as even in cases of complete recovery, the disability remains.

\section{Authors' Contributions}

Bharati Taksande (MBBS, MD): helped in writing the article; Minal Ambade (Medical student): Collected the data by performing the study; Rajnish Joshi (MBBS, MD, MPH): Helped in the statistical part of the study.

\section{References}

1. Rosamond W, Flegal K, Friday G, Furie K, Go A, Greenlund $\mathrm{K}$, Haase N, et al. Heart disease and stroke statistics--2007 update: a report from the American Heart Association Statistics Committee and Stroke Statistics Subcommittee. Circulation. 2007;115(5):e69-171.
2. Easton JD, Saver JL, Albers GW, Alberts MJ, Chaturvedi S, Feldmann E, Hatsukami TS, et al. Definition and evaluation of transient ischemic attack: a scientific statement for healthcare professionals from the American Heart Association/American Stroke Association Stroke Council; Council on Cardiovascular Surgery and Anesthesia; Council on Cardiovascular Radiology and Intervention; Council on Cardiovascular Nursing; and the Interdisciplinary Council on Peripheral Vascular Disease. The American Academy of Neurology affirms the value of this statement as an educational tool for neurologists. Stroke. 2009;40(6):2276-2293.

3. Duparc F, Bocquet G, Simonet J, Freger P. Anatomical basis of the variable aspects of injuries of the axillary nerve (excluding the terminal branches in the deltoid muscle). Surg Radiol Anat. 1997;19(3):127-132.

4. Tsur A, Ring H. Axillary nerve conduction changes in hemiplegia. J Brachial Plex Peripher Nerve Inj. 2008;3:26.

5. Birkner MD, Kalantri S, Solao V, Badam P, Joshi R, Goel A, Pai M, et al. Creating diagnostic scores using data-adaptive regression: An application to prediction of 30-day mortality among stroke victims in a rural hospital in India. Ther Clin Risk Manag. 2007;3(3):475-484.

6. Fitzgerald-Finch OP, Gibson, II. Subluxation of the shoulder in hemiplegia. Age Ageing. 1975;4(1):16-18.

7. Najenson T, Pikielny SS. Malalignment of the GlenoHumeral Joint Following Hemiplegia. A Review of 500 Cases. Ann Phys Med. 1965;8:96-99.

8. Chaco J, Wolf E. Subluxation of the glenohumeral joint in hemiplegia. Am J Phys Med. 1971;50(3):139-143.

9. Moskowitz E. Complications in the rehabilitation of hemiplegic patients. Med Clin North Am. 1969;53(3):541-559.

10. Teasell RW. The painful hemiplegic shoulder. Physical Medicine and Rehabilitation: State of the Art Reviews. 1998;12(3):489-500.

11. Ring H, Tsur A, Vashdi Y: Long-term clinical and electromyographical (EMG) follow-up of hemiplegic's shoulder Eur J Phys Med Rehabil 1993, 3:137-140. 